\title{
Molecular Docking Studies of Dolichin A and B, Pterocarpans from Horsegram (Macrotyloma uniflorum) against HIV Replication Enzymes
}

\author{
L. Rufus Auxilia \\ Research Scholar, \\ Department of Botany and Microbiology \\ Lady Doak College, Madurai
}

\author{
T. Mowna Sundari \\ Research Associate(DBT-BIF) \\ Department of Biotechnology \\ Lady Doak College, Madurai
}

\author{
Rachel Regi Daniel, Ph.D \\ Associate Professor \\ Department of Botany and \\ Microbiology, Lady Doak College
}

\begin{abstract}
Isomeric pterocarpans, Dolichin A and Dolichin B of Macrotyloma uniflorum were docked with the three replication enzymes of HIV (Reverse transcriptase, Protease, Integrase). Analysed results proved that the docking score was high for Protease with Dolichin A (-6.6899) and Dolichin B (6.6944) among Reverse transcriptase and Integrase. AIDS therapies could be scrutinized and /or replaced after the successful inihibitory effect of Dolichin. This study will support the cutting edge of HIV research and drug designing to find out a less side effect causing herbal formulations.
\end{abstract}

\section{Keywords}

Macrotyloma uniflorum, Dolichin A and Dolichin B, AIDS, Docking

\section{INTRODUCTION}

Drug resistance remains a central challenge in HIV therapies. Due to the adverse effects and emergence of drug resistance, an increasing number of patients with HIV infection cannot use the currently approved anti - HIV drugs. Dolichin A and Dolichin B (isomers of 3, 9-dihydroxy-10-(2'-hydroxy-3'methyl-3'-butenyl) are two pterocarpans from Horsegram (Macrotyloma uniflroum) [11]. These pterocarpans constitute the second largest group of natural isoflavanoids with anti HIV activity [8]

The aim of molecular docking is to evaluate the feasible binding geometries of a putative ligand with a target. One of the main goals of drug discovery is the identification of innovative small molecular scaffolds exhibiting high binding affinity and selectivity for the target together with a reasonable absorption, distribution, metabolism, and excretion (ADME) profile [22].

Focus of the present study is to ascertain the inhibitory ability of Dolichin A and Dolichin B from horse gram. Docking was performed for Dolichin A and B with three replicating enzymes of HIV[18]such as Reverse Transcriptase [1][14][17][20][23][24][25],Protease [2] [5] [13] [16] and Integrase [4][19] using GLIDE tool from Schorindger's Maestro. The ADME properties are calculated for Dolichin A and B using Qikprop Tool (Schrodinger, Inc.) designed by Professor William L.Jorgensen [12].

\section{MATERIALS AND METHODS}

\subsection{Preparation of Ligands}

Dolichin A and Dolichin B were retrived from www.lipidmaps.org. The .mol files of ligands were downloaded from the site. The molecules were processed using the LigPrep tool from Schrodinger to obtain the perfect conformation by the addition or removal of hydrogen atoms with respect to the OPLS_2005 forcefield.

\subsection{Preparation of Protein Target}

Targets Reverse Transcriptase 1REV [19] [21] Protease 1EBZ [3][2] and Integrase 1BL3 [7][2] were retrieved from Protein Data Bank (PDB). Water molecules were removed and a single chain was selected between two chains. Generally, all waters (except those coordinated to metals) are deleted, but water that connects between the ligand and the protein are sometimes retained. Problems in the PDB protein structure were repaired by adjusting the protein, metal ions, and cofactors. The structure forming bonds from the ligand or a cofactor to a protein metal were deleted by adjusting the ligand bond orders and formal charges. The minimization was done to restrain the input protein coordinates by a selected RMSD tolerance.

\subsection{ADME Property Screening}

The QikProp tool [12] from Schrodinger helps to screen the molecules for their drug like property by using lipinski's rule of 5[15]. It is a (ADME) prediction program in the Schrodinger's Maestro. It predicts the absorption, distribution, metabolism, and excretion (ADME) physically significant descriptors and pharmaceutically relevant properties of organic molecules.

\subsection{Binding Site Analysis}

The primary binding site analysis was performed using the SiteMap tool from Schrodinger's maestro to identify the active sites on the grid.

\subsection{GLIDE / Ligand Docking}

Grid generated output file was uploaded as an input for Ligand docking against protein prepared targets in GLIDE. SP (Standard Precision) mode was adopted. Flexible docking mode was selected 


\section{RESULTS AND DISCUSSION}

\subsection{Preparation of Ligands}

Figure 1 and 2 represent the 3-D structure of prepared Dolichn A and B.

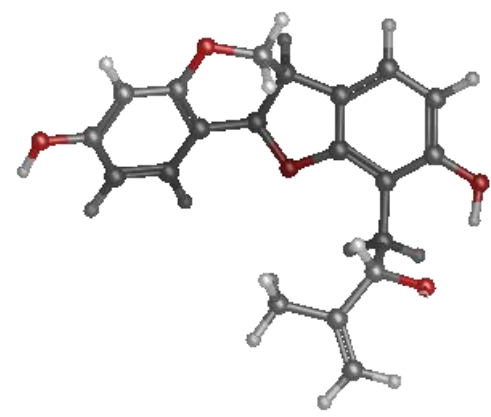

Fig: 1 Chemical structure of Dolichin A

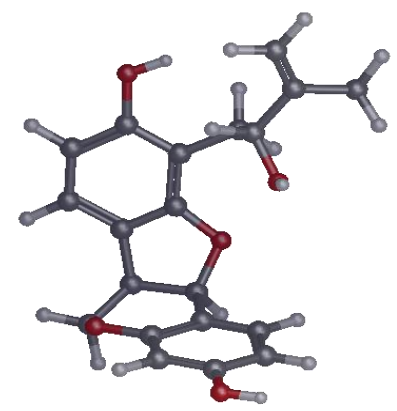

Fig: 2 Chemical structure of Dolichin B

\subsection{Preparation of Protein Target}

The minimized structures of Reverse Transcriptase (1REV) Protease (1EBZ) and Integrase (1BL3) were reviewed to reduce the error in docking.

\subsection{ADME properties of Dolichin A and}

\section{Dolichin B}

Lipinski's rule of five deals with drug likeliness of a chemical compound. ADME screening is important to find out whether a potential chemical compound which could act effectively as drug. Table 1 shows the recommended values of some basic descriptors like molecular weight, $\mathrm{H}$ bond donor, $\mathrm{H}$ bond acceptor, $\log \mathrm{P}$ (octanol/water), Human oral Absorption, CNS etc., The Ligands Dolichin A and Dolichin B perfectly lie on the line of recommended values for the chosen descriptors.

\subsection{Binding Site Analysis}

The active site pockets consisting of hydrophobic, hydrophilic, donor, acceptor and metal binding domains were predicted for all the three targets Reverse Transcriptase, protease and integrase.

3.5 Docking of Dolichin A and Dolichin B The two ligands, Dolichin A and Dolichin B, were selected and their cleaned structures were retrieved from the Ligprep tool. Docking was performed with the three replication enzymes (Reverse Transcriptase, protease and integrase). Table: 2 explain about the docking score, G score and Glide energy mainly and it clearly indicates that,

Protease enzyme has the ability to dock with Ligands Dolichin A and Dolichin B effectively than Reverse Transcriptase and Integrase. The Gscore is calculated for the best pose among the no of poses generated.

Gscore $=a^{*} v d W+b^{*}$ Coul + Lipo + H bond + Metal + BuryP + Rot B + site.

vdW- vanderwall energy; coul- coulomb; ipo- lipophilic contact term; BuryP-penalty for buried polar groups; RotBpenalty for freezing rotatable bonds; site-active site.

\begin{tabular}{|c|l|l|l|l|}
\hline Sl.no & \multicolumn{1}{|c|}{ Properties } & Dolichin A & Dolichin B & \multicolumn{1}{|c|}{ Recommended values* } \\
\hline 1. & Molecular Weight & 340.375 & 340.375 & 130 to 725 \\
\hline 2. & H Bond Donor & 3 & 3 & 0 to 6 \\
\hline 3. & H bond Acceptor & 4.7 & 4.7 & 2 to 20 \\
\hline 4. & Log P & 3.073 & 2.969 & -2 to 6.5 \\
\hline 5. & Human Oral Absorption & 3 & 3 & -1.5 to 1.5 \\
\hline 6. & Percent human oral absorption & 100 & 95.66 & $>80 \%$ is high <25\% is poor \\
\hline 7. & CNS & -1 & -1 & $-2+2$ \\
\hline 8. & VOLUME & 1043.5 & 1037.147 & $500-2000$ \\
\hline 9. & PSA & 80.71 & 78.2 & 7 to 200 \\
\hline
\end{tabular}




\begin{tabular}{|c|l|l|l|l|}
\hline 10. & METAB & 7 & 7 & $1-8$ \\
\hline 11. & QPPCaCo & 962.757 & 738.764 & $<25$ poor, $>500$ great \\
\hline 12. & QPlogBB & -0.754 & -0.811 & $-3.0-1.2$ \\
\hline 13. & QPPMDCK & 474.825 & 356.632 & $<25$ poor, $>500$ great \\
\hline 14. & QPlog Kp & -2.094 & -2.31 & $-8.0--1.0$ \\
\hline
\end{tabular}

Table: 1 The ADME Properties of Dolichin A and Dolichin B using Qikprop tool

*Recommended values - As per the guidelines given in Schrodinger's Maestro Software Suite Manual.

Table: 2 Glide score and Docking Score for the interaction between Dolichin A and B with Reverse Transcriptase, Protease and Integrase

\begin{tabular}{|c|c|c|c|c|c|c|c|}
\hline \multirow{2}{*}{ SI. No } & \multirow{2}{*}{ GLIDE RESULTS } & \multicolumn{2}{|c|}{ REVERSE TRANSCRIPTASE } & \multicolumn{2}{|c|}{ PROTEASE } & \multicolumn{2}{c|}{ INTEGRASE } \\
\cline { 3 - 7 } & & Dolichin A & Dolichin B & Dolichin A & Dolichin B & Dolichin A & Dolichin B \\
\hline 1. & Docking Score & -5.18 & -5.63 & -6.68 & -6.69 & -4.49 & -4.38 \\
\hline 2. & GLIDE Gscore & -5.18 & -5.63 & -6.68 & -6.69 & -4.49 & -4.38 \\
\hline 3. & GLIDE EVDW & -31.54 & -29.94 & -32.96 & -35.68 & -14.64 & -22.66 \\
\hline 4. & GLIDE ECOUL & -5.01 & -6.77 & -14.31 & -14.56 & -14.86 & -12.39 \\
\hline 5. & GLIDE ENERGY & -36.55 & -36.71 & -47.28 & -50.25 & -29.50 & -35.06 \\
\hline
\end{tabular}

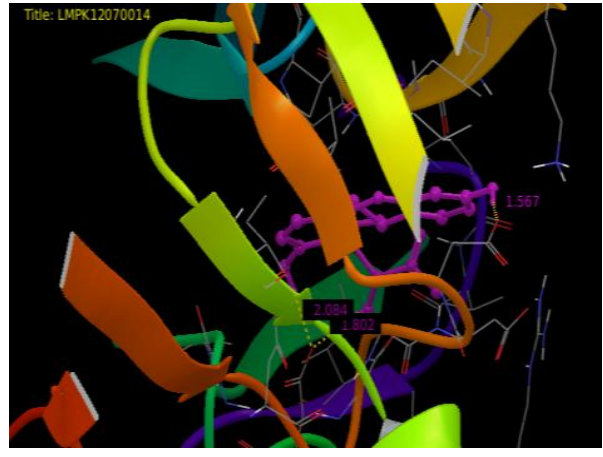

Fig: 3 Protease with Dolichin A

Fig: 3 is the docking complex of Dolichin A and Protease. There are three $\mathrm{OH}$ groups in the Dolichin A chemical structure, two of them are connected to negatively charged Glu in the position of 85 and 87 with the side chain hydrogen bond(Fig:5). The other $\mathrm{OH}$ group is bonded with hydrophobic

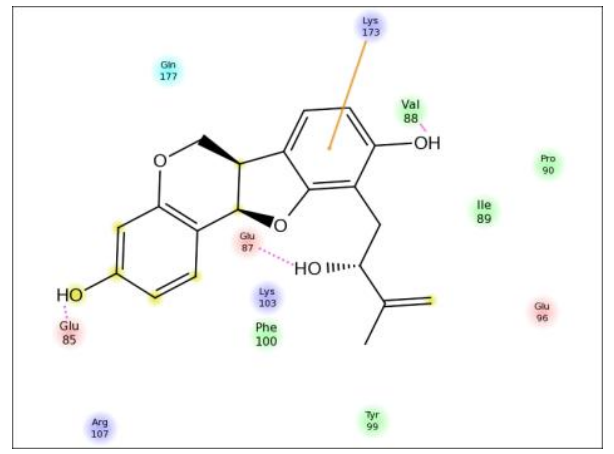

Fig: 5 Interaction of Dolichin A with protease

valine 88 , with the backbone hydrogen bond. Positively charged Lysine 173 has a $\pi$ cationic bond with a benzene ring. The distances of the hydrogen bonds are Glutamine 85- OH (2.084), Glutamine 87-OH (1.802) and Valine $88-\mathrm{OH}$ (1.567). 


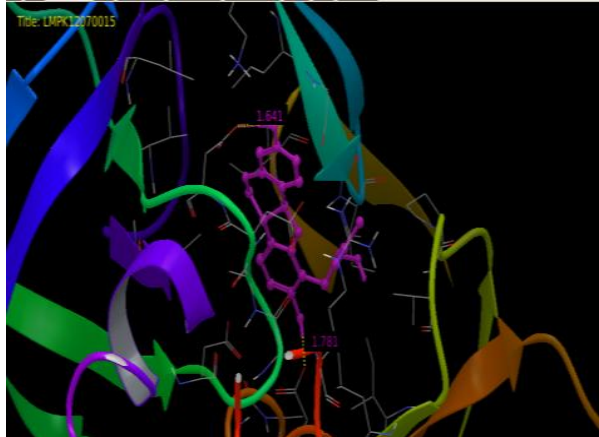

Fig: 4 Protease with Dolichin B

The docking complex of Dolichin B and protease enzyme is exhibited in the Fig : $\mathbf{4}$ and ligand interaction diagram in Fig:6 The two $\mathrm{OH}$ groups are in sidechain hydrogen bond with negatively charged Asp 125 and the other $\mathrm{OH}$ group bonded with ASP 30 with sidechain hydrogen bond. The distances between the hydrogen bonds are 1.781, 1.641 respectively

\section{CONCLUSION}

HIV is a lethal disease spreading all over the world. The drug designing is difficult for HIV as of now, due to the frequent mutation[6][9]in the HIV Several commercially available drugs have developed to prevent HIV from multiplying (making copies of it). It is proved that in many individuals these drugs no longer fight against HIV effectively due to drug resistance. Here we propose an idea of Macrotyloma uniflorum derived lead compounds as an alternate for HIV treatment. Since the insilico study results showed promising docking scores, Dolichin A and Dolichin B can be considered in the drug designing of AIDS.

\section{ACKNOWLEDGEMENTS}

This work was done at DBT - Bioinformatics Infrastructure Facility, Lady Doak College, Madurai. We are grateful to Dr.R.Shenbagarathai, Associate Professor and Head, Department of Biotechnology for her constant support and guidance.

\section{REFERENCES}

[1] Abhik Seal, Riju Aykkal, Rosana O Babu and Mriganka Ghosh (2011). Docking study of HIV-1 reverse transcriptase with phytochemicals. Bioinformation. 5(10): 430-439.

[2] Al-Mawsawi, L. Q., Fikkert, V., Dayam, R., Witvrouw, M., Burke, T. R., Jr.,

[3] Balakrishnan M, Srivastava R.C, Mayank Pokhriyal 2010. Homology Modelling and Docking Studies of HIV Protease. Journal of Biotechnology. 9: 96-100.

[4] Borchers, C. H., and Neamati, N. (2006) Discovery of a small-molecule HIV-1 integrase inhibitor-binding site Proceedings of National Academy of Sciences U. S. A. 103:10080-10085

[5] Brik A \& wong C H,(2003) HIV-1 protease: Mechanism and Drug discovery.Organic and Biomoleculat chemistry, 1: 5-14.

[6] Chen, R., M. Yokoyama, H. Sato, C. Reilly, and L. M. Mansky. 2005. Human immunodeficiency virus mutagenesis during antiviral therapy: impact of drug-

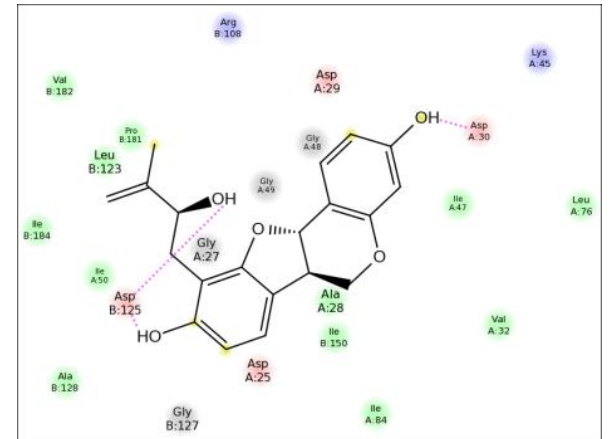

Fig: 6 Interaction of Dolichin B with protease

resistant reverse transcriptase and nucleoside and nonnucleoside reverse transcriptase inhibitors on human immunodeficiency virus type 1 mutation frequencies. Journal of Virology 79:12045-12057.

[7] Cox, A. G., and V. Nair. 2006. Novel HIV integrase inhibitors with anti-HIV activity: insights into integrase inhibition from docking studies.Antiviral Chemistry and Chemotherapy 17:343-35.

[8] Engler T.A., LaTessa K.O., Iyengar R., Chai W., Agrios K (1996). Seteroselective syntheses of substituted pterocarpans with anti-HIV activity, and 5-aza-/5-thiapterocarpan and 2-aryl-2,3-dihydrobenzofuran analogues. Bioorganic and Medicinal Chemistry 4(10):1755-1769

[9] Huang, H., Chopra, R., Verdine, G. L., and Harrison, S. C. (1998) Structure of a covalently trapped catalytic complex of HIV-1 reverse transcriptase: Implications for drug resistance. Science 282, $1669-1675$

[10] Imming P, Sinning C, Meyer A: 2006 Drugs, their targets and the nature and number of drug targets. Nature Review Drug Discovery Oct;5(10):821-34.

[11] Ingham , J.L.,Keen , N.T.,Markham, K. R. and Mulheirn, L.J .(1981) Dolichins A and B, Two Pterocarpans from bacteria treated leaves of Dolichos biflorus. Journal of phytochemistry 20.807

[12] Jorgensen W.L and Duffy E.M.2000. Prediction of Drug solubility from Monte Carlo simulations Bioorganic Medicinal Chemistry Letters 10,1155-1158.

[13] Kohl N E, Emini E A, Schleif W A, Davis L J, Davis J C(1988). Active Human Immunodeficiency virus protease is required for viral infectivity, Proceedings of the National Academy of Sciences USA, 85: 4686-4689.

[14] M.L. Barreca, A.carotti, A.carrieri, A.Chimirri, A.M.Monforte, M.Pellegrini Calace and A.Rao 1999. Comparative Molecular Field Analysis (CoMFA) and Docking Studies of Non- nucleoside HIV RT inhibitors (NNIs).Bioorganic Medicinal Chemistry 7(1999):22832292.

[15] Lipinski CA, Lombardo F, Dominy BM, Feeney PJ.2001.Experimental and Computational approaches to estimate solubility and permeability in Drug Discovery and Development setting. Advance Drug Delivery Reviews 1;46(1-3) 3-26.

[16] Overington JP, Al-Lazikani B, Hopkins AL. 2006: How many drug targets are there? Nature Reviews Drug Discovery. Dec;5(12):993-6. 
[17] Pata J, Stritan W G, Steven G Goldstien and Thomas A.Stiez 2004. Structure of HIV - 1 Reverse Transcriptase bound to an inhibitor active against mutant reverse transcriptases resistant to other non nucleoside inhibitors. Proceedings of the National Academy of Sciences. 101(29):10548-10553.

[18] Rajendra Kumar and Prabha Garg.2010. Active site binding interactions of $\beta$ - Carboline derivative for HIV reverse transcriptase, protease and integrase. International journal of Drug Discovery 2(2): 51-55.

[19] Robinson, W. E., Jr., Reinecke, M. G., Abdel-Malek, S., Jia, Q. \& Chow, S. A(1996) Inhibitors of HIV-1 replication that inhibit HIV integrase. Proceedings of National Academy of Sciences. USA 93, 6326-6331.

[20] Sarafianos G.Stefan, Bruno Marchand, Kalyan Das, Daniel Himmel, Michael A.Parnik, Stephen H.Hughes, and Eddy Arnold.2010. Structure and function of HIV 1 reverse transcriptase: Molecular mechanisms of polymerization and inhibition. Journal of Molecular Biology.23: 385(3): 693-713.

[21] Sengupta D, Verma D, Pradeep K Nair. 2007. Binding Modes, Binding Affinities, and ADME screening of
HIV-1 NNRTI inhibitor Efavirnez and it analogues. Online Journal of Bioinformatics 8(1):99-114.

[22] Sengupta D, Verma D, Pradeep K Nair.2008. Docking MM- GB/SA and ADME screening of HIV-1 NNRTI inhibitor nevirapine and its analogues. In silico Biology, 8,0023 .

[23] Ye XY, Ng TB. 2001 Peptides from pinto bean and red bean with sequence homology to cowpea $10-\mathrm{KDa}$ protein precursor exhibit antifungal, mitogenic and HIV1 Reverse Transcriptase - inhibitory activities. Biochemical and Biophysical Research Communication. 282:424-9.

[24] Ye XY, Ng TB. 2002. Isolation of a new cyclophilin-like protein from chickpeas with mitogenic, antifungal and anti - HIV-1 reverse transcriptase activities . Life sciences.70: 1129-38.

[25] Ye XY, Ng TB.2002 Delandin, a chitinase-like protein with antifungal, HIV-1 Reverse Transcriptase inhibitory activity and mitogenic activities from the rice bean Delandia umbellate. Protein Expression and Purification. 24:

524-9. 